\title{
Detecting Imaging for Neoplastic Liver Masses in Hepatic Patients Who Received Direct Acting Antiviral Drugs
}

\author{
Medhat M. Refaat, Osama T. Galal, Shaimaa A. Taha
}

\begin{abstract}
Department of Radiology, Benha faculty of medicine, Benha University, Egypt.

Correspondence to:

Shaimaa A. Taha, Department of Radiology, Benha faculty of medicine, Benha University, Egypt.

Background: Hepatocellular carcinoma makes up $75 \%-85 \%$ of all primary liver cancers and is the fourth most common cause of cancer related death worldwide. The aim of this study was to screen for hepatocellular carcinoma in hepatic patients who received direct acting antiviral drugs using different imaging modalities. Methods:

Email: This was a cross sectional study included 50 patients who were hepatic and received Direct Acting Antiviral "DAA" and were carried shaimaataha2020@yahoo.com

Received: 6 February 2021 out at radiology department in Elobour Hospital for Health Insurance Accepted: 23 May 2021 after obtaining written informed consent from all patients. Results: There were $31(62 \%)$ male, $19(38 \%)$ female, the mean age 52.28 years old with range (39-65), 35(70\%) urban, 15(30\%) rural. There were (70\%) with duration of illness $\leq 5$ years, $(30 \%)$ with $>5$ years, (100\%) with DAA, $(90 \%)$ easy to treat, $(10 \%)$ difficult to treat. There were $35(70 \%)$ with average liver, 14(28\%) with shrunken liver, 1(2\%) with enlarged liver, 13(26\%) with Bright pattern, 34(68\%) with cirrhotic, and 3(6\%) with coarse bright, the mean P.V diameter 14.68 $\mathrm{mm}( \pm 2.51 \mathrm{SD})$ with range $(9-17 \mathrm{~mm})$. There were $(100 \%)$ with single focal lesion in RT lobe, the mean size was $4.47 \mathrm{~mm}$ with range (3.6-5.6), (33.3\%) isochoric, (33.3\%) with hypo echoic, (33.3\%) with hyper echoic, and (66.7\%) with malignancy. There were 3(100\%) with typical CT enhancement, 1(33.3\%) with P.V thrombosis. None were with affection on liver vascular, no one with intrahepatic biliary radicles, no one with L.N in porta hepatis. Conclusion: Based on our findings, we recommend for close imaging evaluations after DAA in cirrhotic patients.
\end{abstract}

Keywords: Neoplastic; Liver; Masses; Direct Acting Antiviral; DAAs 


\section{Introduction}

Chronic hepatitis $\mathrm{C}$ virus (HCV) infection is one of the most common infections in Egypt especially males which can be responsible for cirrhosis, neoplastic liver masses especially hepatocellular carcinoma (HCC), end- stage liver diseases and other complications such as esophageal varices and portal hypertension (1).

Hepatocellular carcinoma (HCC) is the most common liver cancer and the fifth most common cancer worldwide. It results in between 250,000 and 1 million deaths globally per annum. The number of deaths per year in $\mathrm{HCC}$ is close to that of the incidence throughout the world, which emphasizes the high case fatality rate of this aggressive cancer (2).

Eighty per cent of HCC cases are associated with chronic hepatitis B and C virus infections. The obesity epidemic has resulted in a growing population of patients with non-alcoholic fatty liver disease is a risk factor for cirrhosis and HCC in Egypt (3).

With the rapid development of direct-acting antivirals (DAAs), HCV treatment has become much easier than ever because of the short-term and well-tolerated treatment regimens. Recent studies in real-life clinical practice, including ours, reported that the rates of sustained viral response (SVR) exceeded $90 \%$ for $\mathrm{HCV}$ infection in treatment with sofosbuvir (SOF) plus ledipasvir (LDV) or SOF plus ribavirin (RBV) (4).

Notable improvement in the biochemical parameters of liver function and healthrelated quality of life shortly after treatment. In contrast, recent studies have highlighted the significant impact of early $\mathrm{HCC}$ recurrence However, it remains to be clarified in a large study whether or not the early development of malignant tumors are associated with DAA treatment (5).

As much data as possible on this issue are urgently needed to better manage HCC surveillance in clinical practice. The purpose of this study was to evaluate the short-term risk of neoplastic masses among Egyptian patients with chronic $\mathrm{HCV}$ infection who received direct acting antiviral drugs (6).

Unlike most other cancers, HCC can be diagnosed on imaging studies only without tissue sampling confirmation. Currently, all major consensus groups support the diagnosis of HCC with contrast-enhanced 
multiphasic CT. The accepted modality for neoplastic masses screening is ultrasound. Once malignant tumors are suspected then CT or MRI may be used to confirm the diagnosis. They provide data about liver function, tumor size and number, tumor extent, including vascular invasion and extrahepatic spread, evidence of portal hypertension, and clinical performance status. Tumor proximity to large vessels and main bile ducts (7).

This study aimed to screen for hepatocellular carcinoma in hepatic patients who received direct acting antiviral drugs using different imaging modalities.

\section{Patients and methods}

This study is a cross sectional study, carried out at radiology department in Elobour Hospital for Health Insurance. During the period from July 2019 till January 2020, and included fifty patients who were hepatic and received Direct Acting Antiviral "DAA"

\section{Inclusion criteria:}

1. HCV RNA positive

2. patients $>65$ years old should undergo cardiological assessment prior to therapy by ECG , echocardiology and cardiological consultation

\section{Exclusion criteria:}

1. Child's C cirrhotic patients

2. Platelet count $<50000 / \mathrm{mm}^{2}$

3. Hepatocellular carcinoma except 6 months after intervention aiming at cure with no evidence of activity by dynamic imaging CT or MRI

4. Extra hepatic malignancy except after two years of disease free interval

5. In cases of lymphomas and chronic lymphocytic leukemia treatment can be initiated immediately after remission based on the treating oncologist

6. Pregnancy or inability to use effective contraception

7. Inadequately controlled diabetes mellitus $(\mathrm{HbA} 1 \mathrm{c}>9 \%)$

All patients were subjected to full history taking, complete clinical examination, and Laboratory assessment: Complete blood count, Liver function tests (serum bilirubin "total, direct and indirect ", ALT, AST, total proteins and serum albumin), Kidney function tests (urea and creatinine), Prothrombin time and prothrombin concentration, Viral hepatitis markers (HBV surface antigen, HCV antibody), 
Quantitative HCV-RNA detection using real-time polymerase chain reaction (PCR).

\section{Radiological assessment:}

1.Abdominal ultrasonography (US). Logic P3 for all hepatic patients in the study

2.Triphasic CT for patients with hepatic focal lesions in pelviabdominal ultrasound using Toshiba Alexion 16: Alexion Advanced Edition is a new mid-level 16 Slice CT System which produces high productivity using Toshiba's Exclusive Feature as Industry-Leading Quantum Detector Technology, offering a perfect solution to middle range medical centers and hospitals. It covers all clinical needs of whole body examinations (except cardiac) within a minimum time with an optimization in anatomy visualization, including CT Angiography. Supported by fast accurate Automated Bone Removal, advanced $3 \mathrm{D}$, post processing applications and the latest dose reduction technology integrated from Premium CT Technology for outstanding image quality and patient safety. Using Toshiba's unique "Double Slice Technology", initially developed for the 320-row Aquilion ONE, the Alexion Advance Edition can be configured to generate 32 axial slices in a single rotation using for outstanding spatial resolution and extended flexibility.

An informed verbal consent from all participants was taken and confidentiality of information was assured. An official written administrative permission letter was obtained from dean of faculty of medicine, Benha University hospital manager and Elobour Hospital manager. The title and objectives of the study were explained to them to ensure their cooperation. Permission from the faculty of medicine ethical committee was also obtained, and approval from institutional review board was taken.

\section{Statistical analysis}

Data were fed to the computer and analyzed using IBM SPSS software package version 20.0. (Armonk, NY: IBM Corp) Qualitative data were described using number and percent. The Kolmogorov-Smirnov test was used to verify the normality of distribution Quantitative data were described using range (minimum and maximum), mean, standard deviation, median and interquartile range (IQR). Significance of the obtained results was judged at the $5 \%$ level. The used tests were: Paired t-test: For normally distributed quantitative variables, to compare between two periods. 
Wilcoxon signed ranks test: For abnormally distributed quantitative variables, to compare between two periods

\section{Results}

There were 31 (62\%) males, 19 (38\%) females, the mean age 52.28 yrs $( \pm 6.4$ SD) with range (39-65 yrs), 35(70\%) urban, $15(30 \%)$ rural, $44(88 \%)$ married, $4(8 \%)$ widow, 2(4\%) divorced, 29(58\%) with secondary education, 21(42\%) with university, 46(92\%) Employee, 1(2\%) Unemployed, 3(6\%) housewife, 22(44\%) with low SES, 28(56\%) with middle SES, table 1

There were $35(70 \%)$ with duration of illness $\leq 5$ years, $15(30 \%)$ with $>5$ years, $50(100 \%)$ with DAA, 45(90\%) Easy to treat, $5(10 \%)$ Difficult to treat. table 2

There were $35(70 \%)$ with average liver, $14(28 \%)$ with shrunken liver, 1(2\%) with enlarged liver, 13(26\%) with bright pattern, $34(68 \%)$ with cirrhotic, 3(6\%) with coarse bright, the mean P.V diameter 14.68 $( \pm 2.51$ SD) with range (9-17). figure 1

There were 3(100\%) with single focal lesion in RT lobe, the mean size $4.47( \pm 1.03 \mathrm{SD})$ with range (3.6-5.6), 1(33.3\%) Isoechoic, 1(33.3\%) with Hypo echoic, 1(33.3\%) with
Hyper echoic, 2(66.7\%) with malignancy. figure 2

There were $3(100 \%)$ with typical CT enhancement, 1(33.3\%) with P.V thrombosis. table 3 , there was no one with Affection on liver vascular, no one with intrahepatic biliary radicles, no one with L.N in porta hepatis. table 4

Case 1: (figure 3): Clinical review: 67 years old male patient. He was infected with bilharzial infection and received treatment since 30 years .Recently he discovered infection with virus $\mathrm{C}$ and received Direct Acting Antiviral Drugs DAA for 12 weeks and finished his treatment 4 months ago. Abdominal sonography showed: Cirrhotic liver with mild periportal fibrosis, no focal lesions, patent dilated portal vein, no enlarged LNs in porta hepatis, no intrahepatic biliary radicles and patent hepatic veins

Case 2: (figure 4): Clinical review: 50 years old male patient .He was infected with bilharziasis since 32 years and is infected with virus $\mathrm{C}$ since 4 years and received Direct Acting Antiviral Drugs DAA for 12 weeks and finished his treatment 8 months ago. Abdominal sonography revealed: Cirrhotic average sized liver .patent portal vein, no enlarged LNs in porta hepatis, no 
intrahepatic biliary radicles, and patent hepatic veins. Right lobe shows hypoechoic ill defined focal lesion measures about $3.8 \mathrm{x}$ $4.2 \mathrm{~cm}$. Triphasic CT showed: Right lobe shows intense early enhancement in arterial phase with early washout of contrast lesion which represent typical hepatocellular carcinoma enhancement.

Table (1): Distribution of the studied cases according to demographic data $(n=50)$

\begin{tabular}{|c|c|c|}
\hline & No. & $\%$ \\
\hline \multicolumn{3}{|l|}{ Sex } \\
\hline Male & 31 & 62.0 \\
\hline Female & 19 & 38.0 \\
\hline \multicolumn{3}{|l|}{ Age (years) } \\
\hline Min. - Max. & \multicolumn{2}{|c|}{$39.0-65.0$} \\
\hline Mean \pm SD & \multicolumn{2}{|c|}{$52.28 \pm 6.40$} \\
\hline Median (IQR) & \multicolumn{2}{|c|}{$52.0(47.0-57.0)$} \\
\hline \multicolumn{3}{|l|}{ Residence } \\
\hline Urban & 35 & 70.0 \\
\hline Rural & 15 & 30.0 \\
\hline \multicolumn{3}{|l|}{ Marital status } \\
\hline Married & 44 & 88.0 \\
\hline Widow & 4 & 8.0 \\
\hline Divorced & 2 & 4.0 \\
\hline \multicolumn{3}{|c|}{ Education Secondary } \\
\hline & 29 & 58.0 \\
\hline University & 21 & 42.0 \\
\hline \multicolumn{3}{|c|}{ Occupation Employee } \\
\hline & 46 & 92.0 \\
\hline Unemployed & 1 & 2.0 \\
\hline Housewife & 3 & 6.0 \\
\hline \multicolumn{3}{|c|}{ Socioeconomic level } \\
\hline Low SES & 22 & 44.0 \\
\hline Middle SES & 28 & 56.0 \\
\hline
\end{tabular}


Table (2): Distribution of the studied cases according to HCV history $(n=50)$

\begin{tabular}{lcc}
\multicolumn{1}{c}{ HCV history } & No. & \\
\hline $\begin{array}{l}\text { Illness duration } \\
\quad \text { 5 years }\end{array}$ & 35 & 70.0 \\
$\quad>5$ years & 15 & 30.0 \\
Treatment regimen & & \\
$\quad$ DAA & 50 & 100.0 \\
Patient categories for treatment & & \\
$\quad$ Easy to treat & 45 & 90.0 \\
$\quad$ Difficult to treat & 5 & 10.0 \\
\hline
\end{tabular}

Table (3): Distribution of the studied cases according to CT enhancement and P.V thrombosis $(n=3)$

\begin{tabular}{lll}
\hline & No. & $\%$ \\
\hline CT enhancement & 3 & 100.0 \\
Typical & & \\
P.V thrombosis & & \\
No & 2 & 66.7 \\
Yes & 1 & 33.3 \\
\hline
\end{tabular}

Table (4): Distribution of the studied cases according to different parameters $(n=3)$

\begin{tabular}{llllll}
\hline & No & \multicolumn{3}{c}{ Yes } \\
& No. & $\%$ & No. & $\%$ \\
\hline Affection on liver vascular & 3 & 100.0 & 0 & 0.0 \\
Intrahepatic biliary radicles & 3 & 100.0 & 0 & 0.0 \\
L.N in porta hepatis & 3 & 100.0 & 0 & 0.0 \\
\hline
\end{tabular}


Benha medical journal, vol.38, issue 2, 2021

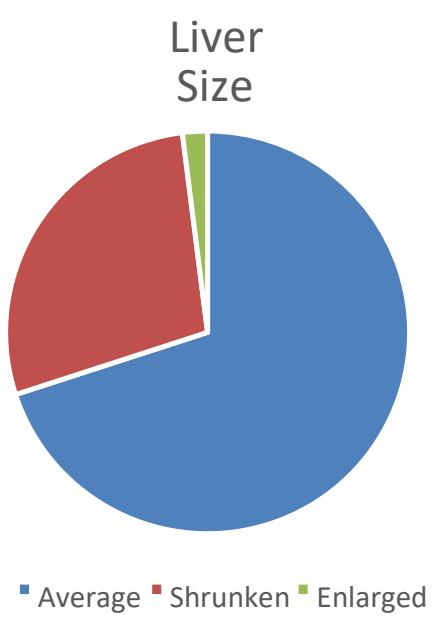

Fig.(1): Distribution of the studied cases according to liver size

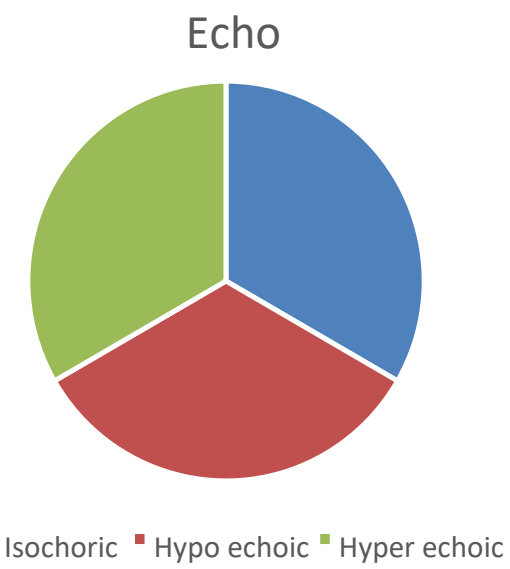

Fig.(2) : Distribution of the studied cases according to Echo 


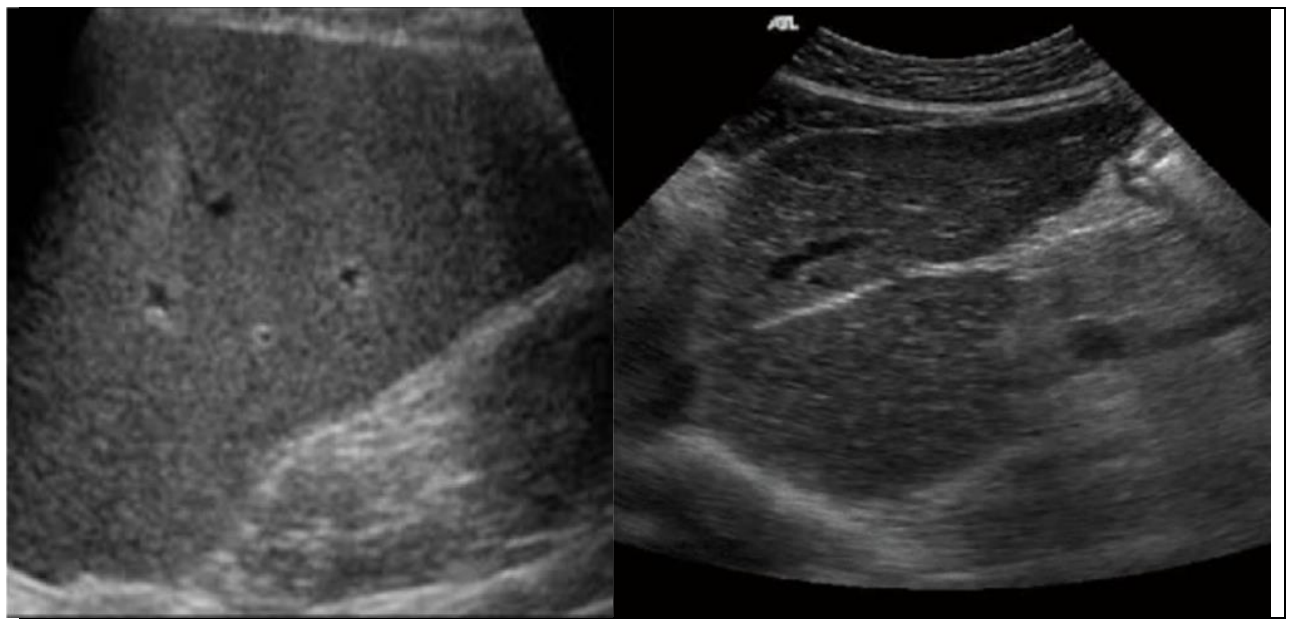

Figure 3: Abdominal sonography: Cirrhotic liver with mild periportal fibrosis. no focal lesions .patent dilated portal vein. No enlarged LNs in porta hepatis. No intrahepatic biliary radicles .patent hepatic veins

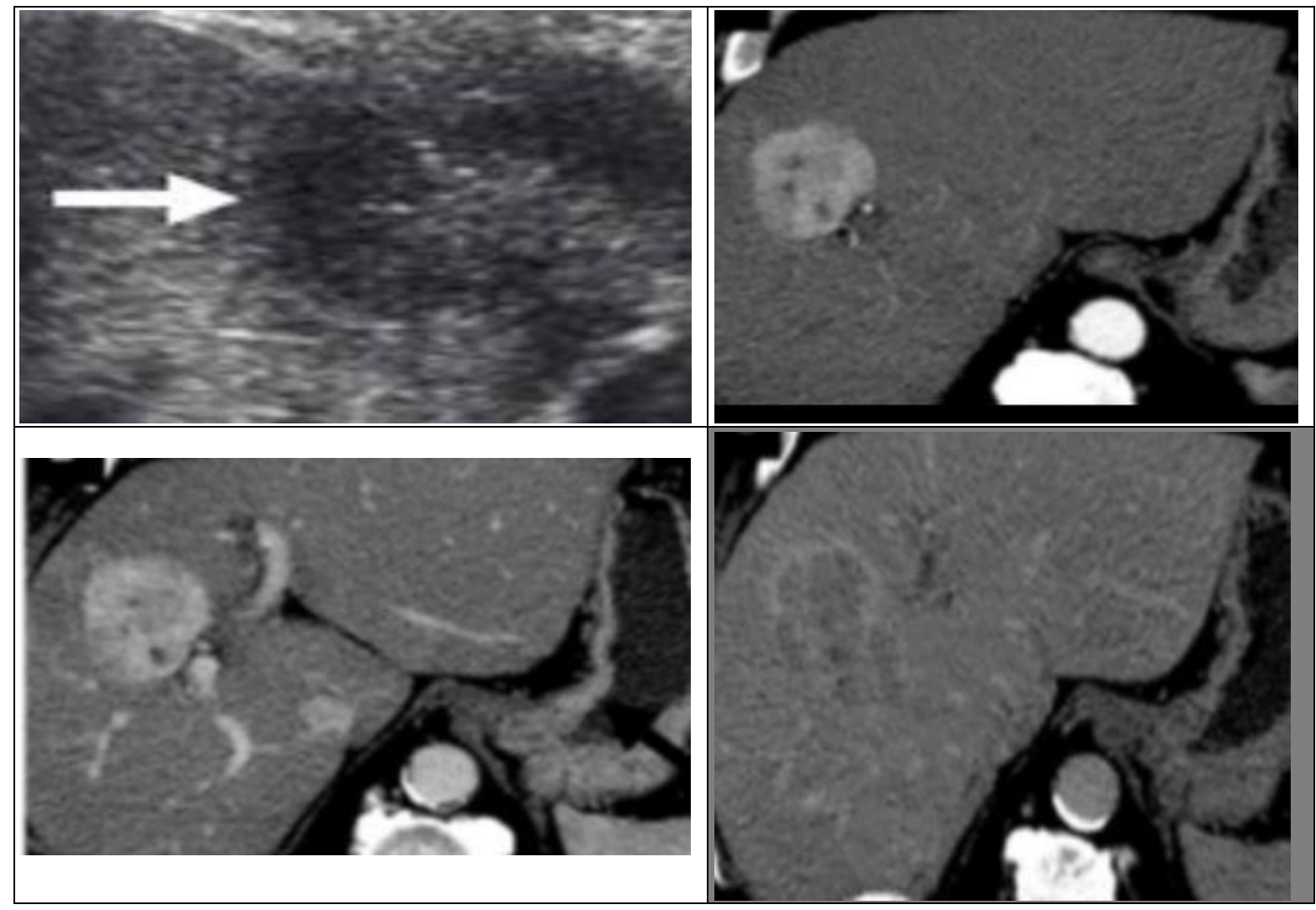

Figure 4: Abdominal sonography: Cirrhotic average sized liver .patent portal vein . No enlarged LNs in porta hepatis . No intrahepatic biliary radicles . patent hepatic veins .Right lobe shows hypoechoic ill defined focal lesion measures about 3,8 x 4,2 cm Triphasic CT:Right lobe shows intense early enhancement in arterial phase with early washout of contrast lesion which represent typical hepatocellular carcinoma enhancement 


\section{Discussion}

The current study showed that there were $35(70 \%)$ with duration of illness $\leq 5$ years, $15(30 \%)$ with > 5 years, 50(100\%) with DAA, $45(90 \%)$ Easy to treat, $5(10 \%)$ Difficult to treat.

In the study done during the post-DAA treatment follow-up, $\mathrm{HCC}$ was diagnosed in 29 of the 344 cirrhotic patients $(8.43 \%$; $95 \%$ CI: 5.93-11.84). Incident de novo HCC occurred in 11 of the 285 patients without previous HCC (3.86\%; 95\% CI: 2.17-6.78) and recurrent $\mathrm{HCC}$ developed in 18 of 59 patients with previous HCC (30.5\%; 95\% CI: 20.2-43.1). The median interval between the end of DAA therapy and diagnosis of HCC was 82 days (range: $0-318$ ): 83 days (range: 0-318) for de novo $\mathrm{HCC}$ and 81 days (range: 0-267) for recurrent HCC (8).

According to study done of 6 months of follow-up after beginning DAA therapy, they reported an HCC occurrence rate of $3.2 \%$ (6). Similarly, another study observed cumulative $\mathrm{HCC}$ incidences after 1 year and 2 years of DAA therapy of $1.7 \%$ and $7 \%$, respectively (9). Additionally, study of 1 year follow up after SVR from DAA treatment reported an HCC incidence of
7.4\%. This rate was higher than the previously reported incidence rate for IFN regimens (1.2\%-1.4\%). Median time for HCC development was 7.6 months after HCV-RNA became non-detectable (10).

In the study in our hands, there is no significant difference between 2 groups as regard $\mathrm{CBC}$, LFT, Serum creatinine, FPG and AFP. (Group A: Baseline \& Group B: After 6 months).

Our results were in agreement with study of 6 months of follow-up and HCC occurrence rate of $3.2 \%$ as they reported that there was no significant difference among both studied groups as regard albumin, bilirubin, platelets and AFP (6).

However, another study revealed that the laboratory results of the studied groups showed statistically significant difference between HCC group and the other groups with respect to hemoglobin level, white blood cells count, platelet count, aspartate aminotransferase (AST), alanine aminotransferase, albumen level, serum bilirubin, international normalized ratio, and AFP (11). 
Different groups have recently reported data on the early occurrence and recurrence of HCC after DAA therapy in patients with compensated cirrhosis. In these reports, the clinical benefits provided by $\mathrm{HCV}$ eradication with DAA regimens were hampered by the accelerated development of HCC, occurring soon after DAA therapy end in a significant proportion of patients. In particular, HCC occurred early in over $3 \%$ of patients without a prior history of liver cancer and recurred early in about $30 \%$ of those who had been successfully treated for HCC, and were disease-free for different periods of time. In addition, the emergence of HCC seemed to be characterized by a more aggressive and faster progression to advanced stages (12).

Vascular invasion is a predictor of recurrence and poor overall survival in HCC. In particular, the presence of microvascular invasion (MVI) is a major risk factor for early HCC recurrence after curative treatment (13).

The current study showed that there were $35(70 \%)$ with average liver, 14(28\%) with shrunken liver, 1(2\%) with enlarged liver, 13(26\%) with Bright pattern, 34(68\%) with cirrhotic, 3(6\%) with coarse bright, the mean P.V diameter $14.68( \pm 2.51 \mathrm{SD})$ with range
(9-17). After 6 months, there were 3(100\%) with single focal lesion in RT lobe, the mean size $4.47( \pm 1.03 \mathrm{SD})$ with range (3.6-5.6), $1(33.3 \%)$ isochoric, $1(33.3 \%)$ with hypoechoic, 1(33.3\%) with hyperechoic,

2(66.7\%) with malignancy. There were $3(100 \%)$ with typical CT enhancement, 1(33.3\%) with P.V thrombosis, no one with intrahepatic biliary radicles, and no one with L.N in porta hepatis.

Our results were supported by study which reported that at the time of $\mathrm{HCC}$ diagnosis, 41 neoplastic nodules were found in 29 patients: a single nodule in 22 patients, two nodules in five, and three or more nodules in two. Maximum nodule diameter was between 10 and $20 \mathrm{~mm}$ in 27 nodules, between 20 and $50 \mathrm{~mm}$ in 13, and greater than $50 \mathrm{~mm}$ in one. Significant differences were found in the presence of these radiological signs in the $\mathrm{HCC}$ nodules developed after DAA therapy in comparison with those that occurred in the past, in the same study population. At least two features of MVI were present in 17/51 (33.3\%) previous HCC nodules, but in 29/41 (70.7\%) newly developed HCCs $(\mathrm{p}=0.0007, \mathrm{OR}$ : 0.21; 95\% CI: 0.08-0.50). This difference was marked even in small HCC nodules (10- $20 \mathrm{~mm}$ in diameter), where MVI was 
present in only $7 / 28(25 \%)$ of the past HCC nodules, but in 16/27 (59.3\%) of the HCC nodules developed after DAA therapy $(\mathrm{p}=$ 0.01, OR: 0.23; 95\% CI: 0.07-0.72) (8).

In another study all patients with HCC occurrence did not show any viral relapse, achieving a SVR. The median diameter of lesions was $33 \mathrm{~mm}$ (range 18-57 mm) (14).

Recent reports have raised the hypothesis that DAA therapy may not reduce the incidence of HCC and, paradoxically, could accelerate the de novo occurrence or the recurrence of $\mathrm{HCC}$ in patients with liver cirrhosis (15). In addition, further preliminary data suggest that HCC developed after DAA therapy may present macroscopic aggressive patterns (16).

Several risk factors have been identified that may increase the risk of $\mathrm{HCC}$ recurrence after DAA treatment, such as high liver stiffness, antiviral treatment failure, history of previous $\mathrm{HCC}$ recurrence, previous HCC shape and stage, AFP level values, des- $\gamma$ carbossi-prothrombin (DCP) > $40 \mathrm{mAU} / \mathrm{mL}$, non-curative procedures (such as TACE), anti-HBc positivity and the time interval between HCC complete response and DAA initiation (17).
In a study done during the follow-up period (median: 17 months), 46 (2.7\%) patients developed HCC. The 1-year cumulative rates of de novo $\mathrm{HCC}$ were $0.4 \%$ and $4.9 \%$ for the noncirrhosis and cirrhosis groups respectively (log-rank test: $\mathrm{P}<0.001)(17)$.

Moreover, another large prospective study found that the 6-mo and 1-year probabilities of HCC recurrence after DAA therapy were $12 \%$ and $26.6 \%$, respectively. Previous history of HCC recurrence and tumour size were the only two independent risk factors for early HCC recurrence. They concluded that the probability of early recurrence in patients who had previously been cured of $\mathrm{HCC}$ remained high, despite $\mathrm{HCV}$ eradication by DAA treatment. This risk was comparable, but not higher than, the risk reported in the literature concerning patients who did not receive DAA treatment (18).

\section{Conclusion}

Neoplastic liver masses especially HCC may occur after receiving of DAA but not conditionally correlated, They seems to be more related to cirrhotic cases more than non cirrhotic. Repeated imaging evaluations are needed after DAA in cirrhotic patients. 


\section{References}

1. Seeff LB. Natural history of chronic hepatitis C. Hepatology. 2002;36(5B):s35-46.

2. Torre LA, Bray F, Siegel RL, Ferlay J, LortetTieulent J, Jemal A. Global cancer statistics, 2012. CA Cancer J Clin. 2015;65(2):87-108.

3. Bugianesi E. EASL-EASD-EASO Clinical Practice Guidelines for the management of nonalcoholic fatty liver disease: disease mongering or call to action? Diabetologia. 2016;59(6):1145-7.

4. Ogawa E, Furusyo N, Nomura H, Dohmen K, Higashi N, Takahashi K, et al. NS5A resistanceassociated variants undermine the effectiveness of ledipasvir and sofosbuvir for cirrhotic patients infected with HCV genotype 1b. J Gastroenterol. 2017;52(7):845-54.

5. Younossi ZM, Stepanova M, Afdhal N, Kowdley K V, Zeuzem S, Henry L, et al. Improvement of health-related quality of life and work productivity in chronic hepatitis $\mathrm{C}$ patients with early and advanced fibrosis treated with ledipasvir and sofosbuvir. J Hepatol. 2015;63(2):337-45.

6. Conti F, Buonfiglioli F, Scuteri A, Crespi C, Bolondi L, Caraceni P, et al. Early occurrence and recurrence of hepatocellular carcinoma in HCV-related cirrhosis treated with direct-acting antivirals. J Hepatol. 2016;

7. Bruix J, Sherman M. Management of hepatocellular carcinoma: an update. Hepatology. 2011;53(3):1020-2.

8. Renzulli M, Buonfiglioli F, Conti F, Brocchi S, Serio I, Foschi FG, et al. Imaging features of microvascular invasion in hepatocellular carcinoma developed after direct-acting antiviral therapy in HCV-related cirrhosis. Eur Radiol. 2018;28(2):506-13.

9. Nakao Y, Hashimoto S, Abiru S, Komori A, Yamasaki K, Nagaoka S, et al. Rapidly growing, moderately differentiated HCC: A clinicopathological characteristic of HCC occurrence after IFN-free DAA therapy? J Hepatol. 2018;68(4):854-5.

10. Cardoso H, Vale AM, Rodrigues S, Gonçalves $\mathrm{R}$, Albuquerque A, Pereira $\mathrm{P}$, et al. High incidence of hepatocellular carcinoma following successful interferon-free antiviral therapy for hepatitis C associated cirrhosis. J Hepatol. 2016;65(5):1070-1.

11. Huang AC, Mehta N, Dodge JL, Yao FY, Terrault NA. Directacting antivirals do not increase the risk of hepatocellular carcinoma recurrence after local_regional therapy or liver transplant waitlist dropout. Hepatology. 2018;68(2):449-61.

12. Reig M, Mariño Z, Perelló C, Iñarrairaegui $M$, Ribeiro A, Lens S, et al. Unexpected high rate of early tumor recurrence in patients with HCVrelated HCC undergoing interferonfree therapy. J Hepatol. 2016;

13. Roayaie S, Blume IN, Thung SN, Guido M, Fiel M, Hiotis S, et al. A system of classifying microvascular invasion to predict outcome after resection in patients with hepatocellular carcinoma. Gastroenterology. 2009;137(3):8505.

14. Rinaldi L, Perrella A, Guarino M, De Luca M, Piai G, Coppola $\mathrm{N}$, et al. Incidence and risk factors of early $\mathrm{HCC}$ occurrence in $\mathrm{HCV}$ patients treated with direct acting antivirals: a prospective multicentre study. J Transl Med. 2019;17(1):292.

15. Kozbial K, Moser S, Schwarzer R, Laferl H, AlZoairy R, Stauber R, et al. Unexpected high incidence of hepatocellular carcinoma in cirrhotic patients with sustained virologic response following interferon-free direct-acting antiviral treatment. J Hepatol. 2016;65(4):856-8.

16. Romano A, Capra F, Piovesan S, Chemello L, Cavalletto L, Anastassopoulos G, et al. Incidence and pattern of" de novo" hepatocellular carcinoma in $\mathrm{HCV}$ patients treated with oral 
DAAs. In: Hepatology. WILEY-BLACKWELL 111 RIVER ST, HOBOKEN 070305774, NJ USA; 2016. p. 10A-10A.

17. Ogawa E, Furusyo N, Nomura H, Dohmen K, Higashi N, Takahashi K, et al. Short_term risk of hepatocellular carcinoma after hepatitis $\mathrm{C}$ virus eradication following direct_acting anti_viral treatment. Aliment Pharmacol Ther. 2018;47(1):104-13.
18. Cabibbo G, Cacciola I, Cannavò MR, Madonia S, Calvaruso V, Petta S, et al. Risk of hepatocellular carcinoma (HCC) recurrence in HCV cirrhotic patients treated with Direct Acting Antivirals (DAAs). Dig Liver Dis. 2017;49(1):e59.

To cite this article: Medhat M. Refaat, Osama T. Galal, Shaimaa A. Taha. Detecting Imaging for Neoplastic Liver Masses in Hepatic Patients Who Received Direct Acting Antiviral Drugs. BMFJ 2021;38(2): 657-670. DOI: 10.21608/bmfj.2021.61764.1380 